\title{
ABOUT ATTITUDES TOWARDS DEATH IN THE LIGHT OF PSYCHOLOGY
}

\section{Argo Moor}

\begin{abstract}
The article observes death-consciousness of contemporary people. The decrease of the transcendence thematic in culture is a probable decrease of death thematic. At the same time unfinished grief causes depression and malfunctions. The loss of someone close will always be dramatic, but is the acceptance of our own mortality agreeing with the finality of our own lives? We could outline several criteria as relationships with relatives, life's significance, health which are connected with the the principle that one's death and afterlife correspond to the life they have lived.
\end{abstract}

Keywords: death, death-consciousness, mourning.

Human experience, by nature, is a whole: subjective and consisting of many aspects. Since this subjectivity and wholeness are perhaps more appreciated in psychology than in any other contemporary stream of thinking, psychology enables us to appreciate remembering one part of the human wholeness experience- the mortality of self.

The problems that repressing this particular part of the whole experience may cause can be illustrated with grief.

In Grief, Verena Kast describes various phases of grief and analyses the problems arising during the mourning process. Unfinished grief causes depression, which often remains a mystery to the mourner. It surfaces in situations that associate more or less directly with the deceased person (death of a stranger, anniversary of death, reaching the same age as that of the deceased, places and actions associated with the deceased). In everyday life, unfinished grief may cause a number of malfunctions. It can lead to the inability of forming new relations; to inadequate substitute relations in attempt to "preserve" the deceased; to adapting the lifestyle of the deceased, although it may not fit the mourner; to various psychosomatic illnesses and, in extreme cases, to suicide (Kast 1998). One remains hanging on to the past. Here Kast postulates that the grief experienced at the loss of a close person and the grief that one falls into at the discovery of his own incurable and fatal illness share the same nature. Kast makes yet another important elaboration: not 
only is one at loss with death, but also with life and other people. Moreover, one loses some of his or her own character traits. All of this calls for the need to mourn them through too in order to continuously relate anew to the changing world (Kast 1998).

However, V. Kast does not highlight one vital aspect: from the deathconsciousness point of view there is no substantial difference between a hopelessly ill and a healthy person - they will both die. The difference is not qualitative but quantitative, in death's probable or expected time of occurrence. We live towards death - every day we draw closer to it and no one can say for sure that he will still be alive tomorrow. Hence, it is inescapable that we accept this reality and learn to live with it. And this, too, is generally achieved through mourning.

If the halt of the mourning process over the loss of a close one can cause depression, psychosomatic illnesses, etc., then to what extent, if any, can similar phenomena be caused by refusing to come to terms with our own mortality? Reactions to accepting death are controversial. On one hand, people wonder, "What's the big deal death is inevitable". On the other, unwillingness to accept the death phenomenon is evident, for example, in the fact that the denial of death generally increases until the age of 55, after which in most cases it begins to decrease to the point of virtual acceptance. Moreover, calm preparation for death is considered of vital importance here, whereas one of its core substances is accepting one's own death. It thus becomes clear that personal mortality has not yet been accepted (see The Results of the Sociological Questionnaire). Psychologists have put forward various defences against death:

\section{1.}

V. Kast proposes that the need for megalomania can be understood as a need for fusing with transcendence. Mortality pictures an individual as trivial whereas in merging with transcendence there is no must to prove one's grandiosity. Likewise, there is no reason to fall into depression when one does not meet the requirements - one is a participant in something big, yet differs from it (Kast 1998).

This proposition is, no doubt, interesting. It enables us to conclude that with the decrease of the transcendence themes in culture, there is a probable decrease of death themes, too, for people cannot tolerate triviality. 
M. Williams sees sadomasochism as a way of conquering the fear of death. Fear of helplessness is characteristic to a sadist (everyone is helpless in the face of death). That fear is compensated with dominant behaviour, where he identifies with death the "unconquerable destroyer". A masochist represents the invincible destroyer, a saviour who liberates him from mortality. M. Williams raises the issue of the repression of death in relationships and concludes that when both partners attempt to overcome death at all cost, they end up in a sadomasochistic relationship where one is the destroyer and the other the destroyed (Williams 1958).

V. Kast proposes that perhaps this is not merely a human problem but also a problem of humanity - to what extent this destructionism originates from such a unilateral identification with death the destroyer (Kast 1998).

\section{3.}

V. Kast considers lust for power a battle with mortality. Loss of power is very painful to overcome - not only influence, money, etc is lost, but with saying goodbye to what is already lost we are reminded of our own mortality. She adds that $\mathrm{H}$. Wilke also presents a thesis whereby it is of vital importance to discuss mortality and the themes of death when analysing people who are suffering from problems of authority (Kast 1998).

4.

V. Kast observes lowering the values or assigning undeserved value to something in relation to the fear of death. In a way, we attempt to gain power by lowering the values - we declare worthless what is important to others. There is also a self-related aspect to it - a person who senses the temporality of life does not conclude hence that he has to start living in the "farewell" mode and consequently escape the temptation to consider life trivial. Assigning undeserved value to things is an attempt to at least do away with the fear of becoming wretched (Kast 1998). 
5.

The disappearance of death's transcendent dimension (e.g. when one identifies merely with death the destroyer) can cause a lifestyle favouring stagnancy and violence (Kast 1998). In extreme cases this may lead to a necrophilic orientation in life - although living, the living are not tolerated. Kast also sees the identification with death the destroyer as one of the causes for depressive illnesses (Kast 1998).

Here we can come to a significant conclusion - not only does the decline of the transcendent dimension cause the fading of the death thematic (due to the fear of pettiness), it also brings on its metamorphosis and distortion (through unilateral identification with death the destroyer). This very distortion validates new efforts of pushing death as a topic into the periphery of culture.

There is a series of more 'innocent' ways of denying death. They are attempts to carry on living through children, creation, works, living memories, the eternal nature, etc (see Death-consciousness). 'Keeping busy against death' or moving on for the sake of it is, according to M. Scheler, a resistance to the rapid transience of everything. Yet another option is to remain on a child's level of development, says Kast (Kast 1998).

One of the most common ways of beating the fear of death is probably dying while living. This is self-destructive behaviour in the broadest sense - destroying the quality of life with a multifaceted arsenal (e.g. drinking, smoking, addictive relationships, consumer mentality, and negligence). When life is made worthless, death is no longer frightening - on the contrary, death has become a saviourredeemer. This raises a serious question about the current tendencies of the contemporary European culture: to what extent is the devaluation (often under the tag of liberalisation) and alienation of values linked with the denial of death?

We should now briefly discuss the positive consequences that accepting the mortality of self can have. Since there is probably no qualitative difference between the grief caused by the loss of a close person and the grief through which we accept our own mortality, we will here, too, draw parallels between the two. Beforehand, how- 
ever, we shall attempt to map the differences of nuances, which I consider significant.

Grief resulting from the loss of someone close will always be more or less dramatic. Emotional bonds do not break by themselves, they require living through. This can also take place before the person's real death, e.g. in the case of a long-term illness. However, under certain circumstances the acceptance of our own mortality can be reached harmoniously. Nevertheless, even then it is not a complete end to death-related anxiety - concern for others remains, concern for the ones who are close and whose wellbeing depends on the person acknowledging death. According to the existentialists, the concern or fear for others always recedes eventually into fear for one's own self, ultimately into the fear of not being.

Here I approve of Emmanuel Lévinas' view whereby the concern for another is also considered an irreducible quality - with such an anxiety there is no concern for oneself (Lévinas 1996). It is believable that such concern for another is more characteristic to someone who has come to terms with his own mortality. In everyday life, however, it manifests mostly in parents' interaction with their children. What then are the positive consequences of embracing mortality?

1. Characteristic to most modern people is a vast outward activity, career-, success- and convenience-orientation etc. The hidden side to such orientation tends to be a rather modest contact with the inner world, individual thoughts, feelings and the bodily sensations. Insufficient touch with the inner world is often apparent through a fragmented and incomplete self-awareness (men are typically out of touch with their feelings, women with their bodily sensations), which disables us to adequately sense our needs. Such segmented understanding of self is undoubtedly restraining to our interest in life and individual nature. In extreme cases it can lead to various psychic or psychosomatic disorders.

The grief caused by losing someone close and the process of acknowledging personal mortality are both likely to cause decline in the contact with the external and an increased contact with the inner world. A moderate growth in touch with the inner world has, no doubt, a balancing effect on a modern human being. It is impor- 
tant though that the withdrawal from the external does not drag on for too long. Similarly, in traditional cultures the duty to mourn is typical, but at the same time mourning for too long is discouraged.

When we lose a close person, our contact with the inner world increases. Perhaps the best example here is that the characteristics, which were previously linked with the lost individual, become our personal possibilities (Kast 1998). Thus, we can talk about the rise of new opportunities and abilities here, too, like in the process of becoming aware of one's own mortality. This is due to the release of a great quantity of mental energy, which had been previously tied up with various defensive positions. This newly released energy may well form the basis for new relationships and possibilities. Deathconsciousness can become a part of self-awareness (Kast 1998) given the context, this is clearly one of the universal outputs here.

2. Acknowledging the loss of a loved one finishes the grief. An important component here is to recognise some significant point in the death event (Kast 1998). Similarly, the process of coming to terms with one's own mortality can be realised at the appearance of a new idea or a new meaning. Kast also analyses the searching disposition as a phase of grief. She comes to the realisation that the searching disposition should not be interpreted merely as compensation - one does not only search for the lost person but also for clarity. A new way of relating to self and the surrounding world is the true object of searching (Kast 1998).

I see the similar effect manifesting where the relations with the close ones are conflicting - seeking justification often belongs to such a lifestyle. Here one is no longer afraid of death. Neither does one feel a direct longing for death as do the elderly or the ones who have lost someone very close. One rather seeks situations where there is a possibility to be killed in one way or another. Having overcome such a situation, one feels brand-new, like being born again. It is as if they have been given the permission to continue living. Thus it is understandable that after the loss of and mourning over someone close, also after the process of acknowledging personal mortality, one begins to differentiate between the important and the trivial. The ripening of a person's values happens mostly through crises, of which the death-related experiences are probably the biggest and have the greatest significance. 
3. Many situations are associated with death: loneliness, old age, loss of a loved one, changes, choices, etc. The acceptance of personal mortality significantly reduces the fear of such situations and makes going through them easier and more harmonious. Presumably, this does not apply to one situation - fear for another. The ones who have accepted their own mortality can only hope that their close and dear ones can come to that too, for they already have. Coping in different situations can also be handled on a more abstract plane - coming to terms with personal mortality makes it easier to deal with various situations containing the fundamental fears (see Death-consciousness).

4. Accepting mortality means agreeing with the finality of our own lives. In that case, it is easier for us to agree with things ending in general. Mortality (termination) is not solely linked with specific situations that actualise death-consciousness - all events in life are started, engaged in and finished. Principally, we can talk about finished and unfinished events in a person's life. Obviously, we can propose different criteria for determining whether an event is finished or not. The simplest criterion classifies these events as unfinished that we could have finished already but which for some reason are still not done. For example, I could have tidied up my room a week ago already; I could have ended or sorted out a relationship two months ago already; I could have fulfilled a promise made to myself a while ago, etc. Another considerable inner criterion by which we can declare an event finished is peace - the feeling of contentment regarding that particular event. Finishing an event presupposes an open and active disposition towards the event and is undoubtedly an effective disposition for life. On the other hand, we can distinguish unfinished events or deeds by the fact that our mental energy remains tied up with them. In a longer perspective, this may lead to the inability of relating continuously anew to the changing world.

It is believable that the inability of finishing acts is partially linked with the denial of our own deaths and the inability to cope with the finality of our lives.

It is important to elaborate the thesis of finishing events from the psychological context into the social context in order to introduce the ethical dimension. From a purely psychological aspect, the is- 
sue of finishing events can be reduced to bringing one's own feelings, desires and needs to an end, expressing them. Such expression may be adequate from the perspective of our inner world (thoughts, feelings, and bodily senses). However, no one lives alone in the world - thus the significance of the question what we express ourselves is equalled by that of the question of how we express ourselves. There is always a witness to one's acts - animals, birds, plants, things, etc. in the traditional cultures; God in the Christian cultures; another human being (if he actually happens to see the act) in the contemporary European culture. At the occurrence of the eyewitness, we can talk about communicative ethics - there is always someone next to or near a person. It is believable that with the withdrawal of communicability (see the analysis of relationships in Self, Soul and Death) the importance of the how we express ourselves principle, and of ethics in general, will gradually diminish.

5. My starting point in evaluating the actualisation of death-consciousness is the axiom that there are no inherently good or bad things, beings, phenomena in the world. There are just different ways of going through events - we are either open or closed in the events. Events in which we hold an open disposition usually start with interest. Interest leads us to the state of openness, the corresponding emotion to which is love. Such events end with satisfaction. These subjectively pleasant emotions are the very reason why we rate this kind of events as good (Sergej Strekalov, oral communication).

Events in which we are closed start mostly with indifference. Indifference is followed by closedness, the corresponding emotion to which is anger. Anger is followed by dissatisfaction (fear, to be more precise). These subjectively unpleasant emotions are the reason why we consider the events, in which we are closed, bad. Events, which we assess negatively, remain to some extent unfinished. Although the event has apparently ended, it is still going on within us - we try to avoid such events and all that relates to it. Consequently, the unfinished events compress our freedom of choice while being the source of our fears. It is clear even without further explanation that life is much more a delight when we are open in various events. Openness, however, presupposes interest in the event. Interest, in turn, depends on certain preconditions. 
Interest links with two important aspects: the external and the internal aspect. Belonging with the external aspect of interest are phenomena which can be joined under the common nominator new new places, people, creation; but also hobbies and risk. On the one hand, it is understandable that a person whose life is full of newness finds relatively easily interest and satisfaction in events. On the other hand, the external aspects of interest are either difficult to control or controlling them requires considerable resources. Since they have no direct link with death-consciousness (with the exclusion of risk perhaps), we can hence proceed without discussing them.

Thus, we shall focus on the internal aspect of interest, which constitutes of four quite easily controllable components:

1. Sufficient quantity of spare energy;

2. Denial of knowledge;

3. Acting for oneself;

4. Acting in a specific form (S. Strekalov, oral communication).

\section{Sufficient quantity of spare energy}

Finding interest presupposes the existence of spare energy. Experientially it is easily understood that we can sense similar situations differently. For example, we come to an unknown wasteland while hiking. It is very likely that in a fresh and agile individual this situation brings forth interest, but rather frightens a tired person. The latter will try to get back to a familiar place as soon as possible. A weary individual will not find satisfaction in events for he wants to rest and recharge his energy supplies. A rested individual, however, is characterised by the need to act (Moreno 1934).

\section{Denial of knowledge}

Here denial of knowledge signifies the positions from where we communicate with that, which we think ourselves as knowing and being acquainted with. For example, leaning on our experience we have the knowledge about citizen $\mathrm{S}$ as always being kind and helpful. Based on this view we go and ask him a favour - we presume that he will do it. If for some reason citizen $\mathrm{S}$ refuses, we are disappointed. The beginning of the event is characterised with indifference manifested in the approach I know he is like that. When citi- 
zen S did not confirm what we knew, anger and eventually dissatisfaction probably occurred. Thus the often-used expression $I$ know in daily life mostly expresses a person's indifference and often refers to closedness towards the given event.

On the one hand, relying on personal experiences and the resultant opinions is natural, but on the other, the world, which we live in, is inconstant and does not always confirm our opinions. People's knowledge is always incomplete (in this case, we have no knowledge of the background to citizen S's refusal). Excessive reliance on personal knowledge may bring about a situation where we project our own fear- or ambition-induced opinions on the surrounding individuals (we invent the reasons for citizen S's refusal). Consequently, our perception of reality becomes distorted. A strong will to depend on the already known may bring about a situation where we attempt to control the surroundings (drawing from our fabricated reasons we try, for example, to manipulate citizen S). Firstly, our effort may fail, causing thus new dissatisfaction. Secondly, such controlling is rather energy-consuming and may, in the end, reduce the first prerequisite of interest, the spare energy.

However, denial of knowledge can bring about real interest towards the situation, thus helping to resolve the situation more effectively and economically.

The I don't know attitude can be evaluated as a learning disposition. Effective learning presupposes openness. It starts from confessing $I$ don't know, which creates interest, the prerequisite of openness. A much less effective learning disposition is a constant comparison of the new material with that, which we know, thus the presumption $I$ know.

\section{Acting for oneself}

People motivate their acts differently. Generally it can be said that one acts for oneself (the reasons are within oneself) or because of others (the reasons lie in the others). Typically, people find more interest and satisfaction in events where they feel that their reasons for participation are personal. When one senses the reasons being other people's dispositions, the acts are often perceived as merely fulfilling duties where the real interest is often missing and satisfaction is smaller or absent altogether. 
While acting for someone else we often get into the state of closedness because we never know precisely what the other person wants. We ask ourselves, what would he/she want? Or, am I doing the right thing? Yet fear (fear of acting incorrectly) is already evident in this process of comparison and we lose touch with the event, i.e. we lose the openness.

I will not tackle the so-called devotional behaviour (e.g. in the philosophy of E. Lévinas), where big diversions from the typical reactions can occur, because it goes beyond the frames of this analysis.

\section{Acting in a specific form}

One's self-perception can be specific or general, abstract. For example, when I am working on something I can decide coming from the general-abstract platform that this task is difficult for me. It is very likely that after making such a decision I quit or finish the task with great difficulty and reluctance.

The same situation may be taken concretely, too - I can describe my thoughts, feelings and senses in detail. For example, while hanging curtains I feel anger, think of my childhood home and sense fatigue in my left shoulder. Such particularisation often produces interest: why do I recall precisely my childhood home? What is it that angers me and why does my left shoulder tire more than the right? Can these bodily sensations and feelings be related? And so on. This means that the appearance and endurance of interest presupposes particularity. Particularity has to do with certain forms. This means that we do not observe ourselves in some general manner but within particular activities, situations and roles. What I am like as a student, a teacher, a father or a child; or when I am angry, tired, interested, etc.

One essential aspect of form is delimitation of time. Setting up a specific timeframe for actions greatly helps the interest to appear and to last. For instance, we can make a decision to take up jogging. We begin with big enthusiasm but with a great likelihood that we will soon quit. The situation can be different if we set up particular time parameters for our decision, e.g. "I wonder what results I will make with six months of practice." Such particularisation of time creates the inclination for interest's endurance and it 
is possible that we complete our set task while also experiencing satisfaction.

\section{Relation of the four prerequisites of interest to remembering the mortality of self}

In everyday life, numerous death-associated phenomena surround us. There are dead chicken and meat of dead pigs on store counters; there is a dried-up, dead tree outside; they talk about the victims of some earthquake on the TV. All that is reality. And this reality reminds us of our own mortality. Since subjectively unpleasant feelings often accompany the actualisation of death-consciousness, people sense and interpret the surroundings selectively, whereas everything death-related is pushed to the background. Such selection requires constant effort, which in most cases remains unacknowledged. The desire to control the surroundings so as to have as little death-consciousness actualising events there as possible requires a lot of energy. Defensive positions towards death are likewise energy consuming.

Thus, we can say that although accepting personal mortality can be a strenuous process requiring a vast amount of energy, it frees us substantially from considerable future exertion. Figuratively we can compare it with the grief one feels at the loss of a close person. Undergoing grief is tiresome and difficult, but at the same time one ends a past relationship through it and gains bigger freedom of choice and more energy for the subsequent life. Therefore, as a result of accepting death-consciousness, one saves energy, which is a crucial prerequisite of interest and openness.

The energy, which was so far spent on denying death (be it through selective reception of information, tentative interpretation, through attempting to control the surroundings, or through defensive positions), is now released for everyday life, relationships and new roles, enriching thus our life.

Nobody knows when they are going to die. Although we may guess or hope that we will live until 80, we do not know for certain even whether we will be alive tomorrow or not. Consequently, neither do we know what will happen next. With the acceptance of this attitude the second aspect of interest is fulfilled - denial of knowledge. 
Since we do not know whether we will live until tomorrow or not, there is no way of knowing anything else about our lives either.

Here we are dealing with a somewhat paradoxical issue, since at the same time, it is also natural to wish to know what comes next - we plan our lives. Thus, it is difficult to say what is harder to bear - a constant and tiring ignorance about the future or a brain-numbing routine. This aspect of interest is directly connected with the first one - the less spare energy we have, the less we tolerate the unawareness regarding the future. This unawareness is most likely to cause fear, turning us into closed persons. Sufficient quantity of spare energy, however, creates a situation where the same unawareness brings forth interest. This paradox is perhaps most expressively exhibited by Ursula K. Le Guin in her book The Left Hand of Darkness (2002). She writes, "The only thing that makes life possible is permanent, intolerable uncertainty; not knowing what comes next."

The general rule is that the more aware we are of our mortality, the more we sense that the life we live is our own - we feel that we make personal choices and are responsible for them.

For instance, I have asked people of different age and sex to make a list of activities they would engage in if they found out they had ten years, one year, one month or one day left to live. There appears a clear tendency that the less time people believe they have been left, the more they do things for themselves. This is most expressively evident with the middle-aged women who go to work and whose spare time is spent on their family life. Making this kind of lists has often been a strong existential experience for them, prompting them to reassess their values at times. Such towards-the-self directed behaviour at the approaching of death is also apparent in a common motif, according to which the dying always tell the truth others' opinions are of no interest to them any longer.

Eventually, the fact of mortality is the time limit of human life, the particularisation of time for our lives. Particularity, however, is again a precondition of interest and openness.

All four premises of interest and thus of openness (sufficient quantity of spare energy, denial of knowledge, acting for oneself and acting in a specific form) are also present in children's games. Chil- 
dren are often energetic and lack specified goals in their games what is going to happen, will become clear during the game. Children play for themselves, not for the pleasure of their parents or neighbours. Moreover, they always play a specific game: shop, birthday, etc.

In my opinion, particularity as an interest producing factor is most expressively evident here, in children's games, for "just being" means just being bored. As soon as particularisation occurs and the form of the game is found - e.g. "let's play the Indians", "let's play school" - , there is also interest - thrill.

With grownups, these attributes appear at indulging in hobbies or at conscious engaging with the inner world (meditation, for instance).

Therefore, we can say that with the disappearance of childlike playfulness people become increasingly closed. The primary playful-open relationship survives through hobbies and can also be found in conscious engaging with the self. It is remarkable that the attributes of playfulness characterise the additional two situations - remembering personal mortality, and love.

6.

Regarding the acceptance of mortality V. Kast also writes that in love, the presence of death is sensed as the moment combining the greatest feeling of living and the sensation of oneness. She says, "It would not be a sensation of oneness if life and death were not standing side by side" (Kast 1998).

I understand love as a feeling descriptive of a person in the state of openness. Openness itself, however, can, be directed towards a particular object (in this particular context the concept of love was discussed in the 5th point). It may also be more intense and directed towards many objects, becoming thus a sensation of oneness (this is the context for the concept of love in the current point). In the extreme cases, openness can become all-inclusive with nothing remaining outside of it. Then we can already talk about uncondi- 
tional love (I shall use this context for the concept of love in the following point).

As seen, love and death belong together in the sensation of oneness (a very common motif in good world literature). Thus, we can state more particularly that in the state of deep love we are aware of the possibility of our death and that of our partner. Therefore, it is also believable that the acceptance of personal mortality eases our arrival at the state of love.

7.

Essentially the same logic is valid for the all-inclusive sensation of oneness - the unconditional love. A strong denial of one part of the oneness experience makes it harder to reach the experience of oneness and vice versa. That is, complying with mortality as probably one of the most problematic components of the oneness experience (at least within the contemporary European culture) we shift closer to the likelihood of that experience occurring.

V. Kast calls this sensation of oneness a symbiosis with something transcendent (Kast 1998). Nevertheless, she does not come to the postulation of one essential attribute of the sensation of oneness. In this sensation of oneness the subject-object opposition (everything is linked with everything) falls apart; likewise, the life-death opposition falls apart. While there is a clear awareness of death (one trait of the sensation of oneness is often a particularly sharpened observance), death is no longer real. In the sensation of oneness, we experience the feeling of immortality.

It is apparent that the feeling of immortality in the sensation of oneness is linked with the qualitatively changed relationship to the self. Logically, this change can be simultaneously expressed as a complete loss of self and as a broadening of self's boundaries to the infinity. When nothing is left outside the boundaries of self, the self has substantially no meaning - the nil in the Eastern philosophy signifies infinity in the Western thought.

The denying/rejecting disposition towards death and that of complying/living with death could be depicted as a spectrum where in 
one extreme there are ruinous/destructive relationships and in the other, love.

In the light of this spectrum, what should one's disposition of life be like? I think the disposition 'prescribed' by death-consciousness consists of beginning and finishing activities - opening up to various events, participating in these events and finishing them. Thus, it would mean being ready for change - readiness to give up even what we have built up with great efforts and care. No doubt, finding such readiness and keeping it is difficult. Here I agree with V. Kast it presupposes leaning on the unity with greater wholes, i.e. leaning on the transcendent background in ourselves; also leaning on creativity, which enables us to relate to the changing world over and over again.

Hence, death-consciousness also 'demands' giving up the feelings of security and safety. What has once been an irreplaceable condition for our development into individuals must now be left behind as an illusion. Indeed, we never know what comes next, how the world will change and how we will change within that world. And living in such unfamiliarity is strenuous.

Consequently, death-consciousness means readiness to give up ourselves - the self. Such letting go can be illuminating but also destructive - in either case it is usually hard. Thus, it is not surprising if people prefer to live denying death, although 'life will then turn against them'.

Here a question about the ideological dimension of psychology arises - about its association with cultural background. Observing the European psychology and psychologists within the framework of three opposing pairs - close to nature vs. urban, traditional vs. modern, collective vs. individual - it is easy to see that they are positioned in one extreme. They represent urban, modern and individualistic cultural type. Is not the thesis whereby the unchanging psychological nature reaches maturity in the early childhood linked with that culture's denial of death?

And did not the bearers of traditional cultures - often the wanderers in solitude in vast wastelands - find psychologically more effective ways, like play, for relating to the unknown and to death, which 
is one of its primary forms of appearance (see 'nature games' in Relation to the Unknown and Death)?

\section{WHAT WOULD THE PRICE OF CHANGES BE?}

I present it as a hypothesis according to which the price of changes is mostly soul-ache - changes are undergone in agony (except the playful variants) and that agony is inescapable. I present this hypothesis in a simplified form leaving out the details that are not crucial for the understanding of the death-related thematic.

The core of the hypothesis is the approach to openness and closedness (finished and unfinished events) by S. Strekalov, a Russian doctorgeneticist, and his definition of the unfinished events' nature.

There are no good things or bad things, there exists only people's openness and closedness in various events. Since in the state of openness one experiences chiefly subjectively pleasant feelings, these events and the associating objects are deemed good. In the state of closedness, one experiences mainly unpleasant feelings and these events along with the associating objects are considered bad. The criterion for finishing events is satisfaction, as noted earlier, whereas in my opinion, the substance of these events is in the fact that the sensations and opportunities, which were during the event associated with the other party, become personal. They become personal sensations that 'nobody can take away' and personal opportunities to the one who has finished the event.

Here we are dealing with an analogy of grief (Kast 1998). To be more precise, it is perhaps in grief that the essence of people's relations to all kinds of events is most organically displayed. But what will come of the unfinished events? According to S. Strekalov, such events 'get stuck' in various parts of body (a strong parallel with Chinese medicine), and the associating information and feelings (which are chiefly subjectively unpleasant) will 'capsulate' there. Although the events are not lived through to the end, the associating memories and feelings fade away. This is due to the formation of a kind of capsule of mental energy in the particular part of the body where the event 'got stuck'. That region of the body has a tendency to form tensions since the mental energy, which is not 
allowed to be expressed through feelings, transfers onto the physical plane. It turns into bodily sensations (pressure, pain, etc.) and in a longer perspective, grows into an illness in the given region. Again there is an apparent analogy with Chinese medicine here, but also with the cognition of psychosomatic illnesses in the contemporary European medical science (S. Strekalov, oral communication).

Humans as whole systems of thoughts, feelings and bodily sensations have three corresponding opportunities to bring the unfinished events to the surface in order to finish them consciously.

The essence of the thought- or information-related opportunity lies in happening to come across a situation similar to or associated with (more or less similar singular elements in different events) the unfinished deed. Here the associating details bring out the past unfinished or repressed event; for example, red rubber boots or foggy weather may remind of a dramatic break-up.

The feelings-related opportunity is essentially a possibility that at the entrance to whatever feeling (except perhaps satisfaction) linked with a current event, the unfinished past experiences associated with these feelings will come to life. For instance, sadness over the ending of some event actualises an earlier break-up with a close person, at which we also felt sadness.

The opportunity relating to bodily sensations is in essence a chance that activating the specific body parts, where the unfinished events and the associating information and feelings 'get stuck', brings the unfinished events to life. The particular body parts are activated through massage or a similar method of applying pressure to them (Strekalov, oral communication).

There is a fourth way as well, which can be used equally in association with thoughts, feelings and bodily sensations. It is a considerable change in body's energy supplies. This change occurs, firstly, with the energy overload, i.e. when an individual is completely rested but is consciously not doing anything - not looking for an object for his or her energy. Additionally, bringing a large amount of oxygen into the body with hyperventilation causes the release of 'excess' energy. Secondly, the change is considerable at the shortage of en- 
ergy, e.g. when an individual is dead-tired, has not slept or eaten, etc. Both, the energy overload and the shortage of energy, bring up the unfinished deeds. The energy overload acts like tidal waters it washes open what has been hidden (capsulated or repressed). The energy deficit, however, works because in order to continue functioning in the worn-out condition, the body needs additional energy since all spare energy is already used up. The additional energy can be drawn from 'around' the repressed events. This is the energy that we use in order not to sense the contact with the subjectively unpleasant memories (information) and feelings present in our body. The price of using that energy, however, is the surfacing of the unfinished deeds and the associating thoughts, feelings and bodily sensations. Again we are compelled to be in the events which were not pleasant for us (S. Strekalov, oral communication).

It is understandable that on the level of everyday life we try to avoid contact with unfinished acts. We avoid situations or singular elements that remind us of these deeds. We avoid subjectively unpleasant feelings and 'choose' our body strategy - posture, the way we walk, we declare certain body regions a taboo, etc (S. Strekalov, oral communication).

Thus, it can be asserted that the boundaries of one's self originate greatly from the surface of the unfinished deeds. It is more difficult to define the extent to which fear of the unfamiliar works as an influencing factor to the setting of the boundaries, since that very unfamiliar may also cause interest.

What constitutes ageing in the given context? The older we get the more unfinished deeds - events, which do not associate with the feelings of peace or satisfaction - accumulate. At the same time, it is believable that the accumulation of unfinished events is rather a progressive predicament than a linear one - the more unfinished events there are, the more particulars of different events start to associate with them: red rubber boots, foggy weathers, specific music, particular seasons, etc. Since these particulars are rather frightening for us, it is probable that the events they occur in will also remain unfinished, thus progressively adding to the number of the frightening elements of events. Thus, we are dealing with the constriction of self-boundaries - the more unfinished deeds there are 


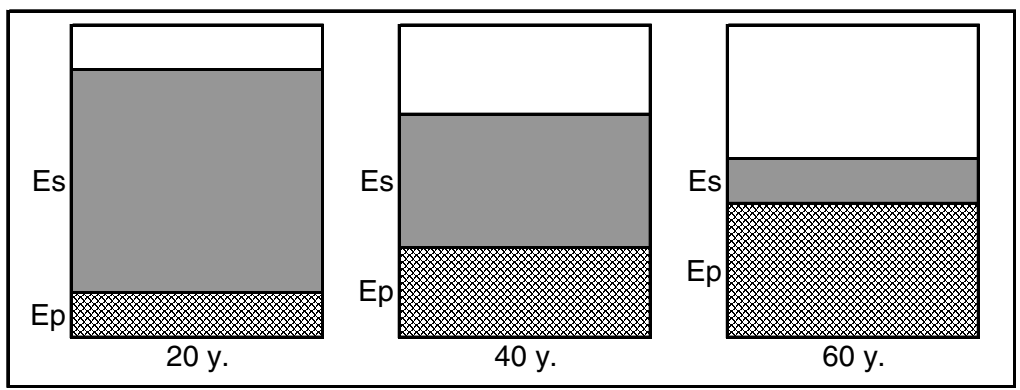

Scheme 1 illustrates schematically the energy balance of a 20,40, and a 60 year old person.

the more will appear, the more fears, the less openness, the more certain insights, etc. there are.

A twenty-year-old individual has already come across some difficulties in life and possesses a few unfinished deeds - on the scheme this is marked with Ep - potential energy. Although this energy exists, its use is avoided lest the thoughts, feelings and bodily sensations associated with the unfinished deeds should surface. The daily activity is plotted largely with the area Es - this is spare energy. After using up the spare energy, the body wants to rest. If one must still do something when tired, 'the mood will go sour'.

With growing older, the overall energetic level drops while the quantity of spare energy diminishes and the amount of unfinished deeds grows. Eventually one starts living in the layer of potential energy for there is no other choice but to get energy from 'around' the unfinished deeds in order to maintain some activity (such an individual can be figuratively pictured as a grumbling old person). Since living within this layer revives unfinished events, the person will start living in memories. Here one selects some two to ten fondest memories from the youth and fixates on them.

\section{CONCLUSION}

Idealistically, ageing or moving towards death actually constitutes finishing events, disposing of them. It takes place due to the dropping energy level. This simplified scheme helps us to understand several 
death-related issues in human life. Drawing also from the aforementioned arguments, we can formulate some consequential conclusions:

1. Boundaries of self are largely determined by the unfinished deeds. We prefer to remain within our boundaries because we avoid contact with the thoughts, feelings and bodily sensations (which in most cases are subjectively unpleasant for us) relating to the unfinished deeds. Alteration and expansion of these boundaries inevitably presumes contact with them and living them through.

2. Liberation from unfinished deeds constitutes agony (thus, agony is a fight for dying rather than a fight against death), the commencement of which is unavoidable during ageing (living in the Ep layer). It is also possible to become consciously free from unfinished deeds (while still living in the Es layer). Here, too, to avoid agony is impossible. However, the released energy remains in our command, producing a basis for new relationships and opportunities.

3. One-sided orientation solely on the pleasant - on what I can and know, on what is familiar to me, etc. - inescapably leads to the increase of the unfinished deeds and to the narrowing of self. Such orientation needs counterbalancing with a disposition of life where we learn to find satisfaction in events that were frightening before (it may also be a conscious work with the inner world).

4. Probably one of the most effective and easiest means to avoid the piling up of unfinished events, the consequent loss in life's worth and of getting rid of the existing unfinished deeds is openness. It is a playful approach to the world and to the self; or at least it counterbalances the motivated, purposeful activity with playful actions.

In a way, playfulness can be perceived as a way of bypassing agony. Agony (letting go) is the harder the more we consider ourselves important or significant. Play rids us from importance but in doing so does not humiliate us. In like manner, remembering death removes our importance, for death is inevitable. Since remembrance of death and playfulness have similar traits, we can say that a deathremembering life is a mode of avoiding or reducing agony.

5 . Based on the above-described scheme we can formulate some important ethical criteria - subjective responses to whether one is living rightly or not. We could outline several criteria such as crea- 
tivity, fears, liveliness, relationships, and success, but in the given context, I would emphasise the following four:

a) Relationships. On a social plane, presumably loneliness corresponds to the internal closedness (the abundance of unfinished deeds), whereas mutually open relationships presumably correspond to openness.

b) Life's significance is drawn from relationships. The feeling that there is a significant meaning to life accompanies open relationships, but in seclusion life's meaning becomes questionable.

c) Health. A great number of diseases are psychosomatic. Unfinished deeds have specific locations in the body. In a longer perspective, the abundance of unfinished deeds leads to worsening of health (in traditional cultures diseases are often perceived as punishment for 'transgressions').

d) Dying. Presumably this scheme also lets us understand several religious motifs, e.g. easy death for the righteous and excruciating death for the wicked, debtors and the renegades; the wicked and the sinners go to hell or purgatory; etc. Such religious motifs can jointly be described with the principle that one's death and afterlife correspond to the life they have lived. The issue of after-death existence is of interest to religions, psychology deals with the current life, though even according to the principles of psychology coming to one's personal hell seems unavoidable.

\section{References}

Kast, Verena 1998. Lein: Psü̈hilise protsessi faasid ja nende ületamise teed [Trauern: Phasen und Chancen des psychischen Prozesses]. Tartu: Johannes Esto Ühing.

Le Guin, Ursula K. 2002. Pimeduse pahem käsi [The left Hand of Darkness]. F-sari. Tallinn: Varrak.

Lévinas, Emmanuel 1996. Eetika ja Lõpmatus: Dialoogid Philippe Nemoga [Ethique et Infini. Dialogues avec Philippe Nemo] . Akadeemia 3, pp. $495-543$.

Moreno, Jacob Levy 1934. Who shall Survive?: A new approach to the problem of human interrelations. Nervous and mental disease monograph series, no. 58. Washington (D.C.): Nervous and mental disease publishing co.

Strekalov, Sergej. Oral communication. 